\title{
Coherent Distributed Techniques for Tactical Radio Networks: Enabling Long Range Communications with Reduced Size, Weight, Power and Cost
}

\author{
Dzulkifli Scherber, Patrick Bidigare, Richard \\ O'Donnell, Matthew Rebholz, Miguel Oyarzun, \\ Charles Obranovich, William Kulp, Daniel Chang \\ Raytheon BBN Technologies \\ Arlington, VA \\ \{d.s.scherber, bidigare, miguel.oyarzun\}@ieee.org \\ \{rodonnell, mrebholz, cobranovich, wkulp, dchang\}
}

abbn.com,

\author{
D Richard Brown III \\ Worcester Polytechnic Institute \\ Worcester, MA \\ drb@wpi.edu
}

\begin{abstract}
This paper addresses the use of distributed coherent communication techniques across a wireless network of tactical radios to enable extended-range communications. Conventional tactical communication systems providing multikilometer terrestrial ranges utilize large and costly manpack or vehicular-mount radios. In this paper we discuss techniques for distributing the transmit functions coherently across a network of small, low-power squad radios to achieve similar ranges. We present a testbed implementation of these techniques and overthe-air demonstrations of coherent distributed transmit communications at $3.5 \mathrm{~km}$ standoff and $1.25 \mathrm{Mbps}$ data rates using radios operating with under $20 \mathrm{dBm}$ of transmit power each.
\end{abstract}

Keywords- Communication equipment; Radio communication; Wireless Networks; Distributed algorithms; Time measurement; MIMO

\section{INTRODUCTION}

Small-unit tactical military communications systems achieve multiple kilometer terrestrial links using large, costly radios, such as those shown in the top three rows of Table 1. To overcome the severe propagation losses associated with terrestrial communications, tactical radios typically employ several Watts of transmit power, necessitating bulky high power amplifiers, cooling apparatus and large batteries to support missions lasting several hours. The size, weight and power $(\mathrm{SWaP})$ of these communications systems impact the health, safety and mobility of the soldiers responsible for carrying them. The costs of these systems further limits their availability.

In recent years, the US armed forces have been equipping individual soldiers with handheld radios such as the AN/PRC154 Rifleman (Table 1 bottom row). These handhelds are intended for individual short-range networked communications

\footnotetext{
* This work was sponsored by the Defense Advanced Research Projects Agency under Army contract W911QX-12-C-0039. Opinions, interpretations, conclusions, and recommendations are those of the authors and are not necessarily endorsed by the United States Government.
}

(generally 100's of meters), and they supplement the longerrange communication systems.

Table 1: Size, weight, power (SWaP) and range of tactical military radios. [1][4]

\begin{tabular}{|l|c|c|c|c|}
\hline & Size & Weight & TX Pwr & $\sim$ Range \\
\hline Falcon III AN/PRC-117G & $6043 \mathrm{~cm}^{3}$ & $5.44 \mathrm{~kg}$ & $5 \mathrm{~W}$ & $>4 \mathrm{~km}$ \\
\hline SINCGARS RT-1523 & $3007 \mathrm{~cm}^{3}$ & $3.5 \mathrm{~kg}$ & $5 \mathrm{~W}$ & $>4 \mathrm{~km}$ \\
\hline JTRS AN/PRC-155 & $4211 \mathrm{~cm}^{3}$ & $6.35 \mathrm{~kg}$ & $20 \mathrm{~W}$ & $>10 \mathrm{~km}$ \\
\hline $\begin{array}{l}\text { JTRS AN/PRC-154 } \\
\text { Rifleman Radio }\end{array}$ & $524 \mathrm{~cm}^{3}$ & $0.9 \mathrm{~kg}$ & $2 \mathrm{~W}$ & $>2 \mathrm{~km}$ \\
\hline
\end{tabular}

In this paper, we describe techniques by which softwaredefined tactical networking radios can be modified to enable a local network of radios to achieve communications ranges comparable to those of much larger and more costly systems. As we will demonstrate, by coordinating their transmissions, a network of tactical radios can match or exceed the performance of a single conventional long-link radio [5].

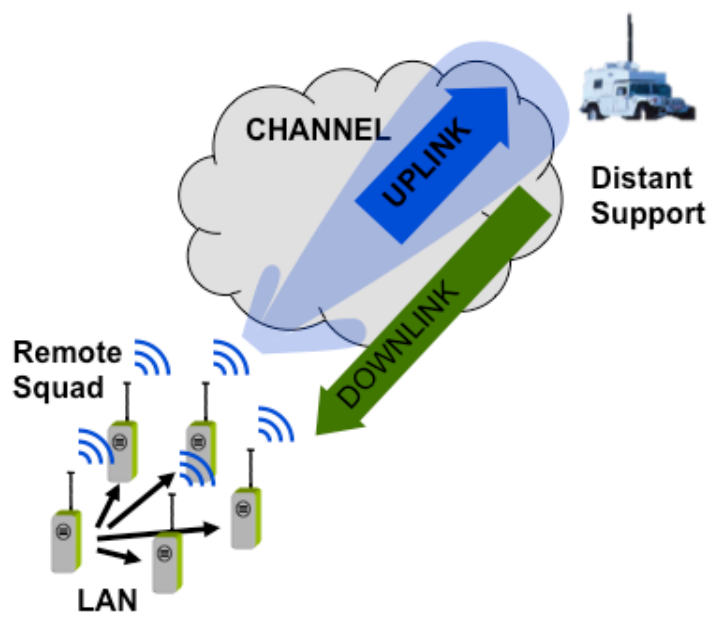

Figure 1: Coherent distributed communications enables a network of radios to extend their range by coordination the transmission of a common message such that, after propagation the signals arrive simultaneously and in-phase at the intended receiver. 
In Figure 1 a squad equipped with tactical networking radios uses its wireless local area network (LAN) to distribute a common message. The distributed radio array transmits this common message over the uplink channel to a remote receiver at the distant support element. The remote receiver is equipped with a low-latency, low data rate feedback downlink to the distributed radio array. The receiver provides transmit compensation coefficients to each of the radios in the distributed array so that the individual transmitted signals arrive simultaneously and in-phase, interfering constructively. Squad radios are assumed to have a wireless local area networking capability along with a separate radio slice used for the coherent distributed long-uplink communications. These two functions may be implemented using separate radio slices and modems in different bands or using a time slotting to share a single radio slice and modem.

The technology required for distributed communications has been maturing rapidly and is now ready for serious consideration in military applications. The theory behind coherent distributed transmit communications has been addressed in [5]-[9], related research for coordinated transmit nullforming has been explored in [10] and [11], and related research has been introduced for distributed receive communications in [12]. Experimentally, over-the-air, distributed time synchronization necessary to support targeted coherent transmissions has been demonstrated [13] and shortrange, stationary, over-the-air tests on SDR platforms have been conducted [14]. This report documents what the authors believe to be the first multi-kilometer demonstration of coherent distributed communications.

Section II provides a terse description of the technique (mathematical exposition appears in [7] and [9]) and a description of the feedback latency requirements to achieve coherence. Section III describes two over-the-air field experiments of distributed transmit beamforming (tones only) and full coherent distributed communications at multikilometer ranges. Sections IV and V discuss the operational benefits of coherent distributed communications and some potential military applications.

\section{PhenOMENOLGY AND TECHNICAL APPROACH}

\section{A. Coherent Distributed Transmissions}

Received power is the product of the total transmit power and total antenna array gain, both of which grow linearly with the number of participating nodes, causing power-on-target to grow quadratically. For communications, this greatly increases the communication ranges or greatly decreases the required per-radio $\mathrm{SWaP}$ for a given range. Figure 2 shows a $5 \mathrm{X}$ available range increase while maintaining a $5 \%$ link outage probability when increasing from a single transmitter to 30 coherent transmitters.

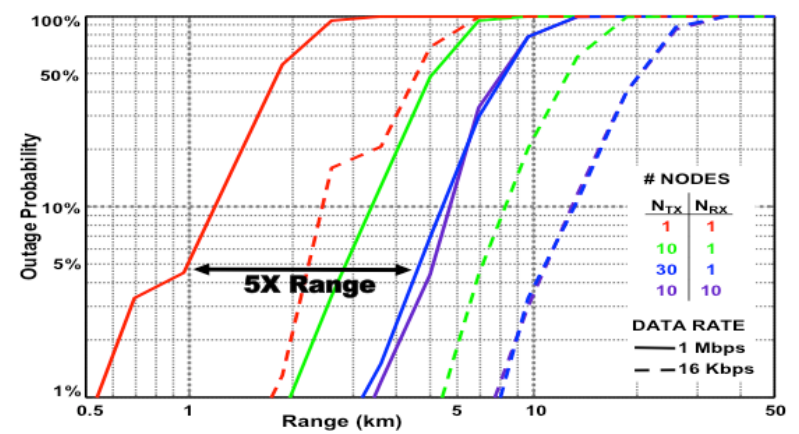

Figure 2: Simulated outage probability vs. communications range for 1,10 and 30 radios using independent Rayleigh channels with variances given by a Longley Rice urban propagation model at $1.8 \mathrm{GHz}, 1 \mathrm{~m}$ antenna heights and $1 \mathrm{~W}$ EIRP.

The coherent distributed transmission technique coordinates a set of networked radios to transmit a common waveform such that the radio frequency carrier waves and the message bearing waveform constructively interfere as they arrive at the desired receiver. The coordination comes in the form of precise scheduling of each transmission to account for each radios independent local oscillator and independent propagation path to the receiver. Figure 3 depicts the time sequence of three transmitters coordinating so their transmissions constructively interfere by arriving at the destination receiver at the same time.

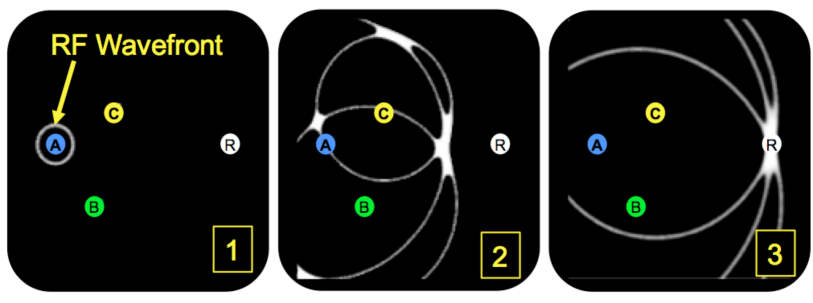

Figure 3: This time sequence shows the fundamental principal of coherent transmission. Three transmitters (A, B, and C) are on the left and one receiver (R) is on the right. Transmitter A must transmit its wavefront before B and C because its propagation time is greatest. Timing the transmissions precisely allows the wavefronts to arrive simultaneously at the receiver.

A feedback loop is used to maintain coordinated transmission across all radios, each with an independent local oscillator and independent motion. The receiver makes a measurement of the carrier-level timing offset for each transmitter during short dedicated channel sounding timeslots (Figure 4: red, yellow and green) that are sent back to the transmitters over the feedback link (blue). These timing offset measurements are incorporated into a higher-order state space estimate of the channel using a Kalman filter framework. The Kalman filter provides channel (timing offset) predictions that can be pre-compensated for during common beamforming intervals (purple). This Kalman filter implementation allows highly non-stationary but predictable channels (large LO offsets or Doppler shifts) to be accommodated. 


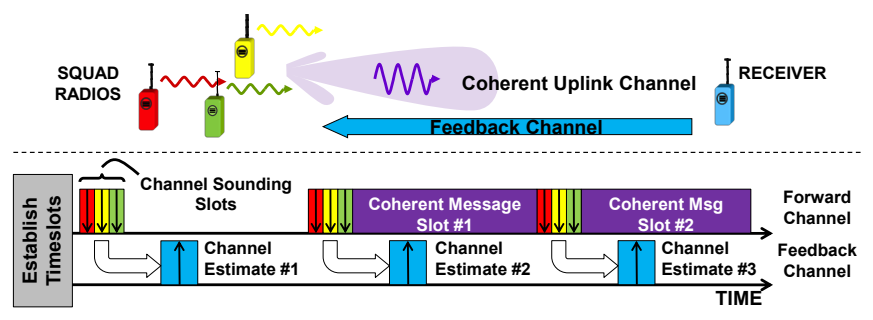

Figure 4: Sounding, feedback and beamforming timeslots implemented in our coherent distributed communications demonstrations.

\section{B. Maintaining Coherence with Motion}

The offset prediction accuracies required for achieving coherency were derived in [9] and are depicted in Figure 5. Achieving $80 \%$ of ideal coherency requires an RMS timing error below $1 / 12$ of a carrier period. For example, at $465 \mathrm{MHz}$ the filter predictions must be accurate to $179 \mathrm{ps}$ while at 2.4 GHz the RMS offset estimate must be within 35ps.

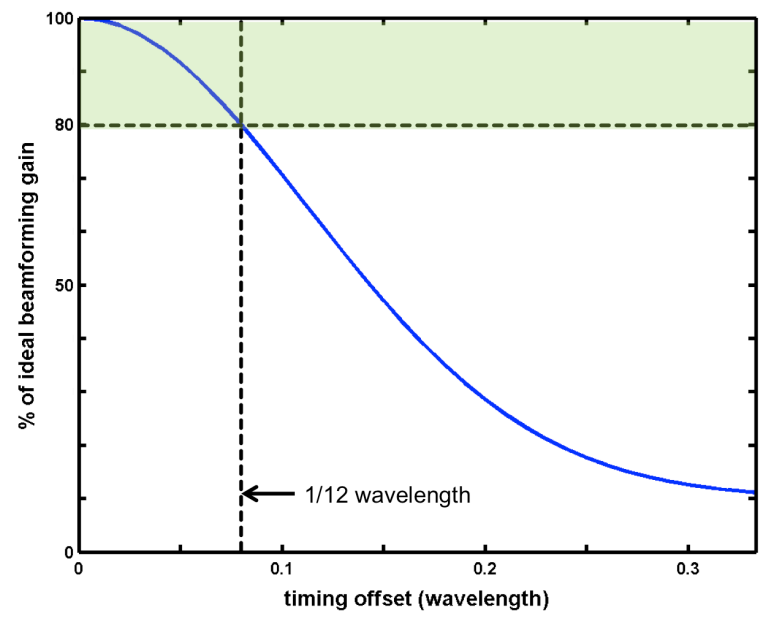

Figure 5: Relationship between waveform timing, relative to carrier frequency, and theoretically achievable coherent beamforming gain.

The predictability of the radio motion critically affects the required feedback latency for a particular carrier frequency. Figure 6 delineates the feedback latency / carrier frequency combinations for which $90 \%$ or better coherency can be achieved for radios experiencing independent sea-state 4 motion. Although sea-state 4 corresponds to highly nonstationary motion with large absolute displacements, the random component of the motion is very low frequency with almost all of its spectral content below $1 \mathrm{~Hz}$. As a result, the Kalman filter is able to accurately predict the channel and coherency can be achieved with large feedback latencies even at very high frequencies. Figure 7 shows coherency vs. latency and frequency for worst-case manned aircraft vibration. In this case, the PSD of the motion has spectral components in the 100 's of $\mathrm{Hz}$ and very tight feedback latencies are required in order to achieve coherence.

For pedestrian motion a feedback latency of $30 \mathrm{~ms}$ to $60 \mathrm{~ms}$ is required to maintain $>90 \%$ coherence when transmitting at or below $\mathrm{L}$ band.

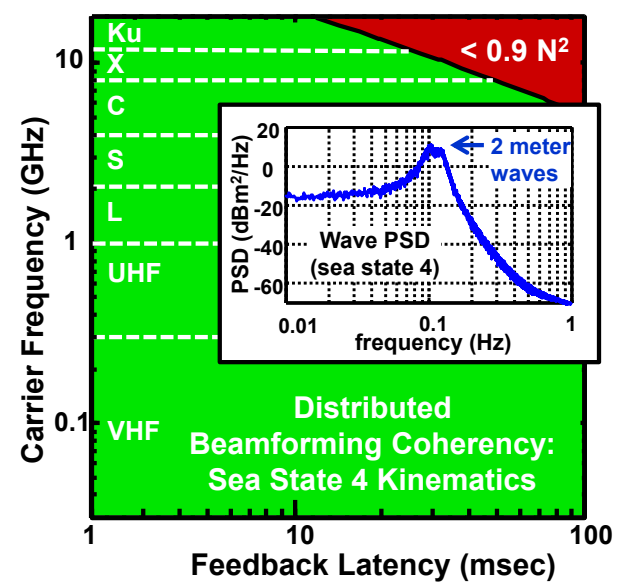

Figure 6: Coherency (Red: $<90 \%$, Green $>90 \%$ ) vs. feedback latency and carrier frequency for radios experiencing independent motion consistent with sea-state 4 wave dynamics.

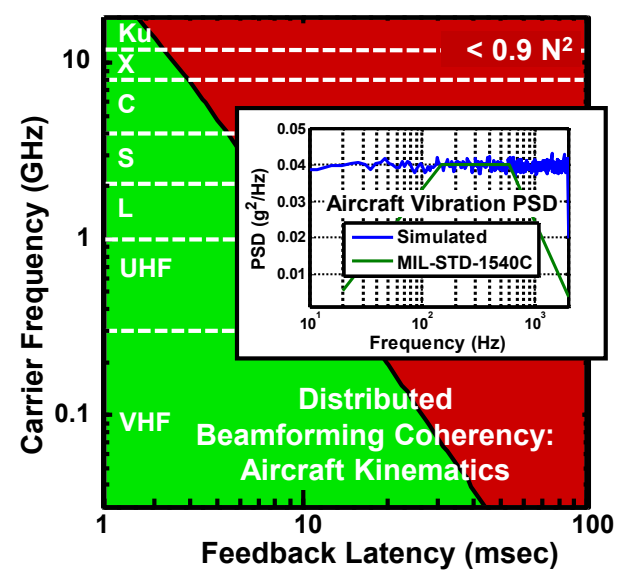

Figure 7: Coherency (Red: $<90 \%$, Green $>90 \%$ ) vs. feedback latency and carrier frequency for radios experiencing independent motion consistent with worst-case (MIL-STD-1540C) manned aircraft vibration.

\section{DEMONSTRATION AND RESUlts}

To demonstrate the performance of distributed transmission, BBN constructed 14 testbed radios using primarily COTS components and conducted two field testing campaigns in November 2011 and January 2013 to demonstrate distributed transmit beamforming and coherent distributed communications respectively.

In November 2011, an over-the-air demonstration of distributed transmit beamforming method over a $1.0 \mathrm{~km}$ link was conducted near Mercersberg, PA. Ten transmit nodes were placed at the top of a slope with line-of-sight to a target receiver. Each transmitter broadcast a tone, with proper realtime phase and frequency corrections, such that it would interfere constructively with tones from the other transmitters at the receiver. Each node could be independently enabled or disabled during the collection.

Figure 8 shows the effect of incrementally adding beamforming nodes to the group, then the effect of disabling the time/frequency alignment. Of particular interest is how quickly the nodes lose synchronization when predicted 
corrections from the Kalman filter are not maintained, as well as the difference in received power when comparing coherent to incoherent transmissions.

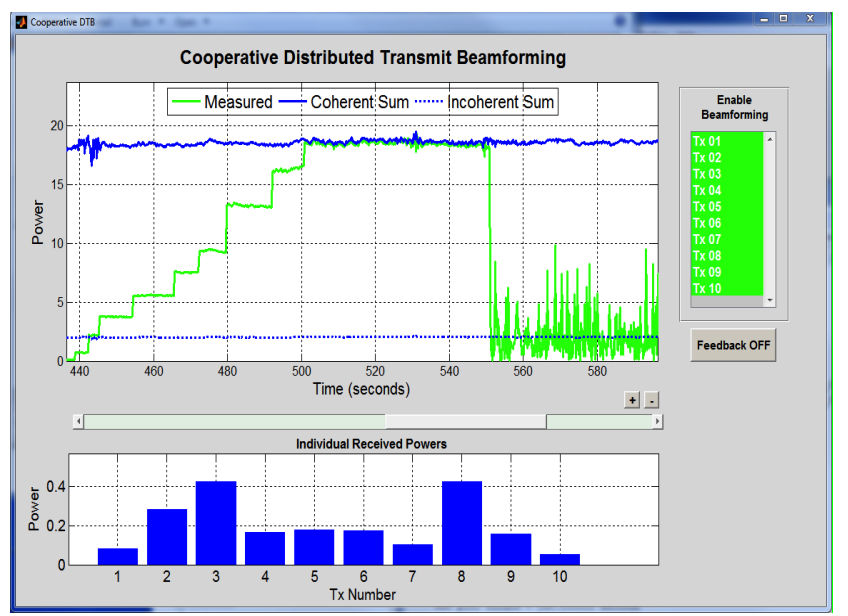

Figure 8: Bottom pane shows individual received node powers while transmitted powers from each radio was the same, showing a diversity of propagation channels to the receiver. Top pane shows ideal coherent sum (blue) vs. actual received power (green) as the 10 nodes are sequentially activated between 420 and 500 seconds. Feedback updates are deactivated at 550 seconds illustrating difference between coherent and incoherent transmission.

Distributed communications tests to demonstrate the ability of distributed transmit communications to reduce the transmit power required and the potential range increases were performed in Delaplane, VA. Figure 9 shows an overhead view of the field test layout. Each thick line represents one of the three ranges that were tested, and at each range the transit power was varied to test the full transmit power scale of the testbed hardware.

At each range, three frequency bands were tested. The tested bands were 200, 480, $920 \mathrm{MHz}$. All of the links were line-ofsight in a rural environment. At each site, five transmitters were placed in an arbitrary formation within $15-30 \mathrm{~m}$ of each other. The receiver was left at a fixed location. Positions were surveyed using GPS.

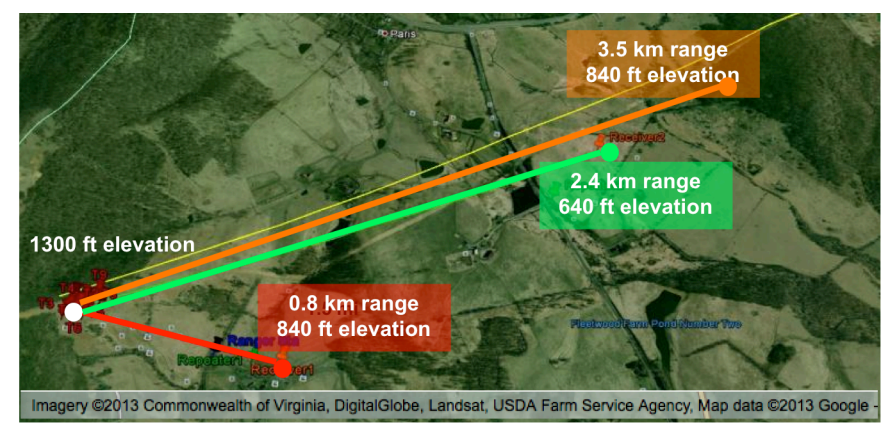

Figure 9: Satellite view of the testing site at Sky Meadows State Park. Three ranges were tested, ranging from $0.8 \mathrm{~km}$ to $3.5 \mathrm{~km}$ line-of-sight links in a rural environment.

To expedite development, Wi-Fi was used as a backchannel link rather than developing a custom feedback link. The communications protocol used a rate $1 / 2$ forward error correcting code based on the DVB-S2 standard with a QPSK symbol constellation and a symbol rate of $1.25 \mathrm{Msps}$. This produced a data rate of approximately $1.25 \mathrm{Mbps}$. Tests were conducted for both stationary transmitters and transmitters experiencing pedestrian motion.

Figure 10 shows the over-the-air results at the $2.4 \mathrm{~km}$ range in the $920 \mathrm{MHz}$ band. The transmitters were swept through the full range of their transmit powers in $2 \mathrm{~dB}$ steps. At each transmit power, block error rates were calculated for each of the 5 individual SISO links as well as the $5 \times 1$ distributed MISO link. The combination at each power-level was exercised five times to give an effective block error rate for each instance.

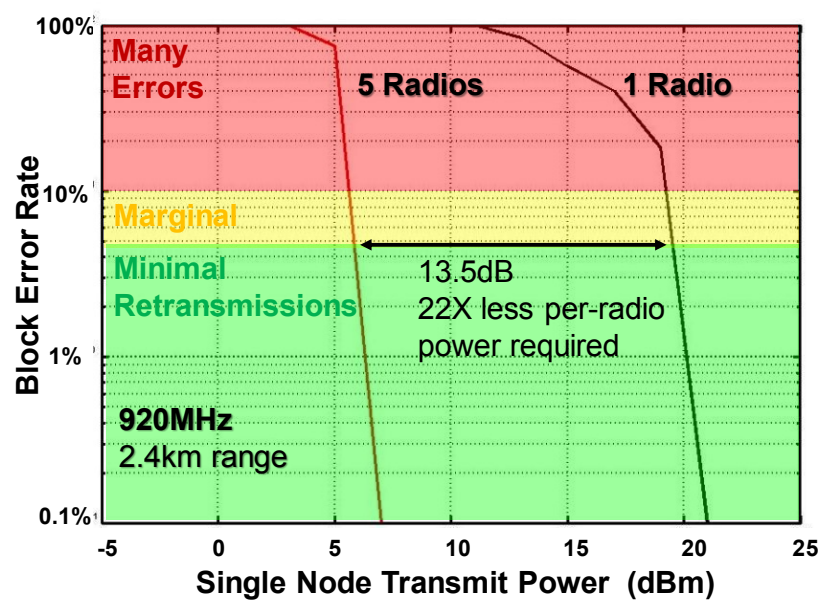

Figure 10: Waterfall plot generated using over-the-air data collected during the January 2013 field test, showing a $13.5 \mathrm{~dB}$ reduction in required per-node transmit power when using five transmit nodes vs. a single transmitter.

The MISO diversity advantage over SISO is evident in the much steeper slope of the MISO waterfall curve.

\section{BENEFITS}

The central benefits of coherent distributed communications are $\mathrm{SWaP}$ reduction, range extension and improved communications reliability.

\section{A. Reduce $S W a P$}

One potential benefit to implementing distributed coherent communications is in the weight and power requirements of tactical radios. To achieve multiple kilometer ranges, current tactical radios have to operate with many Watts of EIRP, requiring correspondingly large power amplifiers. This, in turn, requires larger batteries. Since distributed coherent communications results in quadratic growth of power at the receiver, a single large radio can be replaced with a networked array of much smaller radios, each operating with a fraction of a Watt of EIRP. Ten smaller radios, acting in concert, could perform the function of an equivalent larger radio in terms of measured power at the receiver while providing the additional benefit of significantly smaller volume and weight. 


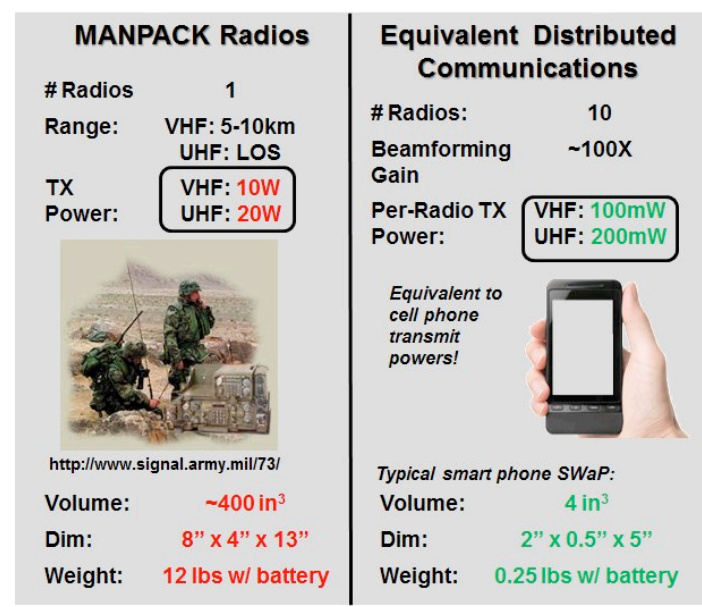

Figure 11: Distributed communications achieves comparable ranges with a $\sim 50 \mathrm{X}$ reduction in per-radio volume and weight.

\section{B. Extend Range}

The extended range benefits of the use of coherent distributed communications are illustrated in Figure 12. These include the increase in terrestrial communications ranges over comparable single radios. Utilizing existing handheld radios, advertised communication ranges of up to $2 \mathrm{~km}$ are achievable. Implementing distributed coherent communications on those same radios would allow 10 radios to extend the ranges out to $6+\mathrm{km}$ while 30 radios could achieve ranges to $10+\mathrm{km}$.

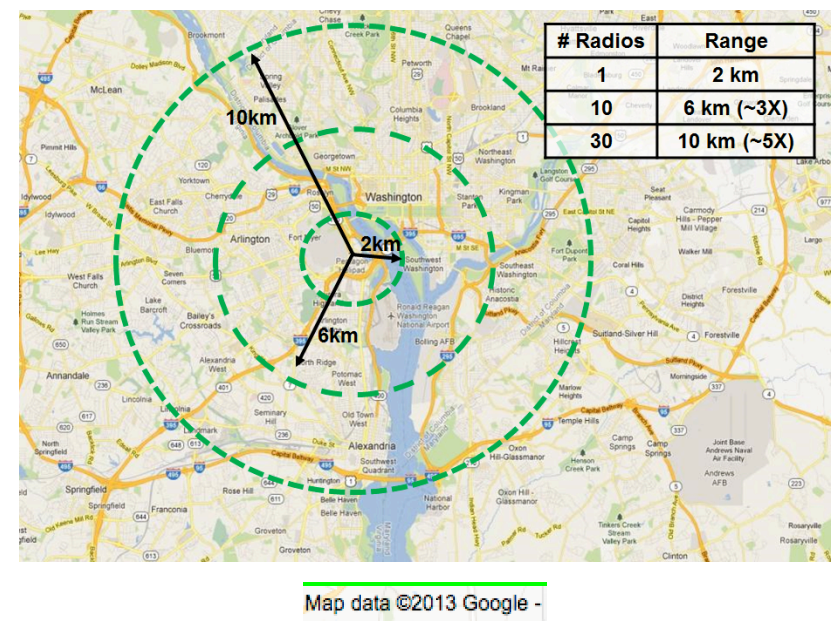

Figure 12: Example of potential terrestrial communications range increases using coherent distributed radio arrays.

\section{Increase reliability}

In addition to the increase in communication ranges, the increased number of radios provides additional tactical benefits. Having multiple radios allows for more signal path diversity, helping to mitigate the effects of multipath and shadowing. Moreover, having a coherent communications capability helps eliminate the reliance on only a few assets and eliminate single points of failure.

\section{APPLICATIONS}

Coherent distributed transmissions provides an enabling technology for several important military applications. A distributed coherent communications capability implemented across networks of handheld or small form factor (HMS) tactical radios has the potential to extend reachback operating ranges by a factor of five or more without requiring any additional communications equipment. Distributed coherent communications has the potential to extend squad-to-squad communications to ranges beyond $10 \mathrm{~km}$.

The technology has the potential to benefit a number of other military applications, as well, where smaller, lighter, long range distributed communications are important. As illustrated in Figure 13, these applications range from the longer range communications at the squad and platoon levels, to higher mobile SATCOM data rates and increased range or data rate communications for UAVs and UGS systems.
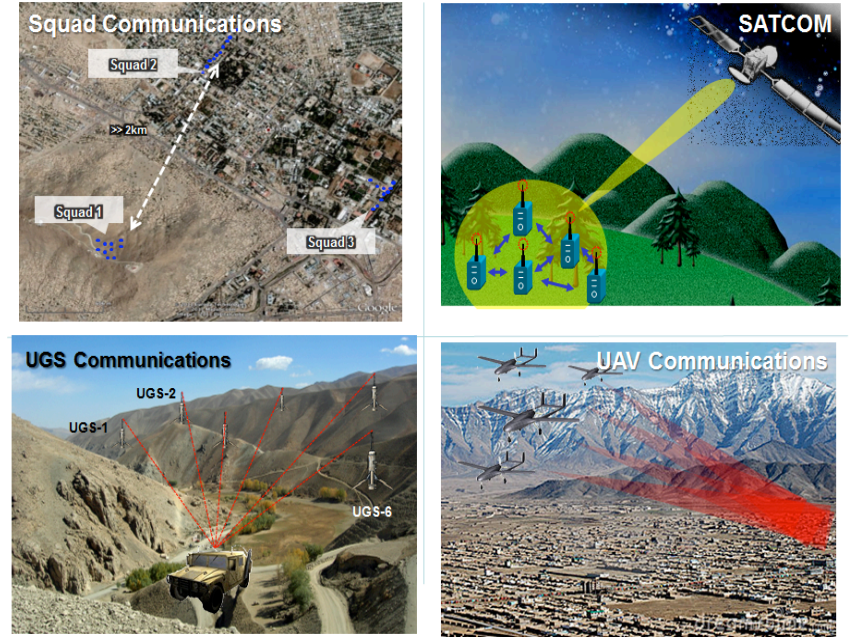

Figure 13: Coherent Communications can benefit a number of different military applications including tactical inter-squad and inter-company communications, SATCOM, UGS and UAV communications.

\section{CONCLUSIONS}

Distributed communications techniques leverage groups of cooperating radios and advanced signal processing to provide large gain increases over equivalent single radios. With distributed transmission, the power at a receiver increases quadratically with the number of transmitters. This quadratic growth in power may be used to enable longer range, higher data rates, and lower size, weight, and power.

Raytheon BBN Technologies has demonstrated over-the-air distributed communications at over $1 \mathrm{Mbps}$ at a range of $3.5 \mathrm{~km}$ using prototype hardware. Coherent distributed communications technology has been proven to be technically feasible, and offers significant promise for many important military applications.

\section{REFERENCES}

[1] Harris. (2012, Dec). Specifications for AN/PRC-117G(V)I(C) [Online]. Available:http://rf.harris.com/media/AN-PRC-117G WEB tcm269017.pdf

[2] ITT Excelis. (2011). SINCGARS RT-1523 Radio [Online]. Available:http://www.exelisinc.com/solutions/SINCGARS-RT1523/Pages/default.aspx

[3] General Dynamics. (2013). AN/PRC-155 Manpack Radio [Online]. Available:http://www.gdradios.com/ANPRC-155.cfm 
[4] General Dynamics. (2013). AN/PRC-154 Rifleman Radio [Online]. Available:http://www.gdradios.com/ANPRC-154.cfm

[5] Yang, J.; Brown, D.R., III, "The Effect of Channel State Information on Optimum Energy Allocation and Energy Efficiency of Cooperative Wireless Transmission Systems," Information Sciences and Systems, 2006 40th Annual Conference on, vol., no., pp.1044,1049, 22-24 March 2006.

[6] Yang, J.; Brown, D.R., III, "The Effect of Receiver Diversity Combining on Optimum Energy Allocation and Energy Efficiency of Cooperative Wireless Transmission Systems," Acoustics, Speech and Signal Processing, 2007. ICASSP 2007. IEEE International Conference on , vol.3, no., pp.III-493,III-496, 15-20 April 2007.

[7] Mudumbai, R.; Madhow, U.; Brown, R.; Bidigare, P., "DSP-centric algorithms for distributed transmit beamforming," Signals, Systems and Computers (ASILOMAR), 2011 Conference Record of the Forty Fifth Asilomar Conference on, vol., no., pp.93,98, 6-9 Nov. 2011.

[8] Bidigare, P.; Madhow, U.; Mudumbai, R.; Scherber, D., "Attaining fundamental bounds on timing synchronization," Acoustics, Speech and Signal Processing (ICASSP), 2012 IEEE International Conference on , vol., no., pp.5229,5232, 25-30 March 2012.

[9] Brown, D.R.; Bidigare, P.; Madhow, U., "Receiver-coordinated distributed transmit beamforming with kinematic tracking," Acoustics, Speech and Signal Processing (ICASSP), 2012 IEEE International Conference on , vol., no., pp.5209,5212, 25-30 March 2012.
[10] Brown, D.R.; Madhow, U.; Bidigare, P.; Dasgupta, S., "Receivercoordinated distributed transmit nullforming with channel state uncertainty," Information Sciences and Systems (CISS), 2012 46th Annual Conference on , vol., no., pp.1,6, 21-23 March 2012

[11] Brown, D.R.; Bidigare, P.; Dasgupta, S.; Madhow, U., "Receivercoordinated zero-forcing distributed transmit nullforming," Statistical Signal Processing Workshop (SSP), 2012 IEEE , vol., no., pp.269,272, 5-8 Aug. 2012.

[12] Brown, D.R.; Ni, M.; Madhow, U.; Bidigare, P., "Distributed Reception with Coarsely-Quantized Observation Exchanges," Information Sciences and Systems (CISS), 2013 47th Annual Conference on , vol., no., pp. , 20-22 March 2013

[13] F. Quitin, U. Madhow, M. M. U. Rahman, R. Mudumbai, "Demonstrating distributed transmit beam- forming with software-defined radios," Proc. 2012 IEEE International Symposium on a World of Wireless, Mobile and Multimedia Networks (WoWMoM 2012), San Francisco, CA, June 2012.

[14] Bidigare, P.; Pruessing, S.; Raeman, D.; Scherber, D.; Madhow, U.; Mudumbai, R., "Initial over-the-air performance assessment of ranging and clock synchronization using radio frequency signal exchange," Statistical Signal Processing Workshop (SSP), 2012 IEEE , vol., no., pp.273,276, 5-8 Aug. 2012. 